\title{
A Continuum Theory of Crack Shielding in Ceramics
}

Division of Engineering, Brown University, Providence, RI 02912
A phenomenological constitutive model is proposed which aims at describing the overall effect of microfracture in ceramics. Based on this model, the asymptotic stress, strain, and displacement fields at the tip of a stationary macroscopic crack are determined in closed form. The near-tip stress-intensity factor is computed and observed to be significantly smaller than the applied stress-intensity factor even for moderate amounts of damage.

\section{Introduction}

Certain classes of ceramics are known to undergo extensive microcracking confined to a process zone surrounding macrocrack tips (Hoagland et al., 1973; Claussen, 1976; Wu et al., 1978). Under these conditions, the processes at the crack tip are screened from the remote loads by the intervening microcracks and a fracture toughness enhancement results (Evans, 1984). Microcracks develop at grain boundary facets mainly as a result of residual stresses generated during cooling and of applied tensile stresses (Fu, 1983). The net effect of the microcracks is to render the material more compliant. Under increasing loads, the microcracks already present in the material remain confined to their respective facets and their size is essentially unaltered. Thus, further elastic degradation comes about as a result of increasing microcrack density and not microcrack growth. Eventually, the number of available nucleation sites which are favorably oriented with respect to the applied tensile loads is exhausted and a saturation stage is reached in which the material does not undergo further damage.

A first attempt at quantifying the crack shielding effect has relied on computer simulation (Hoagland and Embury, 1980). However, an analytical treatment of the problem has proven elusive in part due to lack of adequate material characterization. In this study it is assumed that the length scale over which the singular fields dominate is large compared with the characteristic microcrack size and the mean distance between the microcracks, so that an asymptotic analysis can be based on the effective overall properties of the continuum. Ideally, one would like to have a description of the effective behavior based on micromechanics and a detailed knowledge of its dependence on relevant micromechanical parameters, such as grain size. However, this entails the determination of the ag-

Contributed by the Applied Mechanics Division for presentation at the 1987 Applied Mechanics, Biomechanics, and Fluids Engineering Conference, Cincinnati, $\mathrm{OH}$, June 14-17, 1987, of the American Society of Mechanical Engineers.

Discussion of this paper should be addressed to the Editorial Department, ASME, United Engineering Center, 345 East 47th Street, New York, N.Y. 10017 , and will be accepted until two months after final publication of the paper itself in the Journal of Applied Mechanics. Manuscript received by ASME Applied Mechanics Division, February 12, 1986; final revision, May 22, 1986.

Paper No. 87-APM-9. gregate effect of dense populations of strongly interacting microcracks, possibly with preferred orientations, distributed over a three-dimensional heterogeneous elastic medium. Even under strong simplifying assumptions, this problem poses consideration difficulties (Horii and Nemat-Nasser, 1983; Kachanov, 1985). Thus, to make progress one has to resort to phenomenological models. In Section 2, one such model is proposed which aims at describing the effective behavior of a material undergoing progressive distributed damage and exhibiting a saturation stage. The possibility of a strong damageinduced elastic anisotropy is taken into consideration. Some of the ideas involved have been taken from constitutive models proposed for other progressively fracturing materials such as concrete (Ortiz, 1985).

In Section 3 the near-tip singular fields for a stationary crack are determined in closed form. Then, Rice's $J$-integral (Rice, 1968) is used to relate the stress-intensity factor at the crack tip to the amplitude of the remote $K$ field. It is found that small amounts of damage can result in a substantial reduction of the stress intensity factor and thereby bring about a toughening of the material. This situation is in sharp contrast to transformation toughening which only comes into effect for a growing crack (Budiansky et al., 1983).

\section{Effective Constitutive Behavior of Progessively Fracturing Materials}

A phenomenological constitutive model is presented below which aims at describing the overall effect of microfracture in ceramics. In this work, processes resulting in permanent strains as well as rate effects are neglected. The model is predicated upon the following assumptions. Stresses and strains are assumed to be linearly related according to

$$
\epsilon_{i j}=C_{i j k i} \sigma_{k l}
$$

where the elastic compliances $C_{i j k l}$ are regarded as internal variables which evolve as a result of damage processes taking place at a microstructural level. A threshold is postulated below which no further damage can occur. For the class of materials under consideration, the onset of damage is assumed to occur when the maximum tensile stress $\sigma_{1}$ reaches a critical value $\sigma_{c} \geq 0$, i.e., 


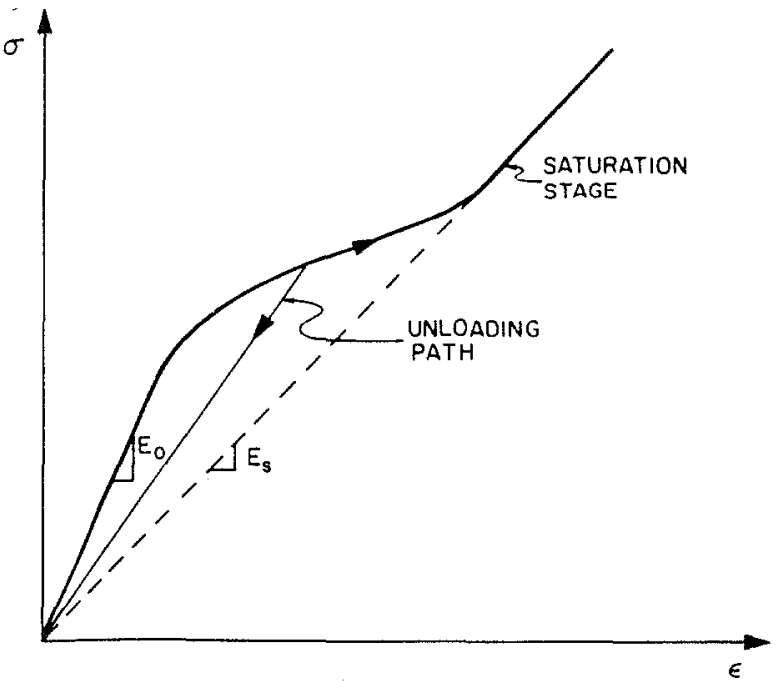

Fig. 1 Uniaxial tension stress-strain curve showing an initial elastic range and a saturation stage

$$
\phi\left(\sigma, \sigma_{c}\right) \equiv \sigma_{1}-\sigma_{c}=0
$$

The direction of incremental damage is given by a damage rule

$$
\dot{C}_{i j k l}=\dot{\lambda} n_{i} n_{j} n_{k} n_{l}
$$

where $\mathbf{n}, \sqrt{n_{i} n_{i}}=1$, is the direction of maximum tensile stress and the multiplier $\lambda$ can be regarded as an effective added flexibility due to damage. Implicit in equation (3) is the assumption that the newly nucleated microcracks tend to be preferentially oriented normal to the direction of maximum tensile stress and thus most loss of stiffness occurs in that direction. Similar ideas were used by Hutchinson (1983) to estimate the macroscopic steady creep-rate of a material exhibiting powerlaw creep and simultaneously undergoing creep-constrained grain boundary cavitation.

The evolution of the critical stress $\sigma_{c}$ is assumed to be governed by a damage rule of the type

$$
\dot{\sigma}_{c}=h\left(\sigma_{c}\right) \dot{\lambda}
$$

for some modulus $h\left(\sigma_{c}\right)$. In this simple model the dependence of $h$ on $\sigma_{c}$ can be determined from the uniaxial tension stressstrain curve alone. Finally, the damage and loading-unloading criteria can be expressed in Kuhn-Tucker form as the requirement that the constraints

$$
\phi \leq 0, \quad \dot{\lambda} \geq 0, \quad \phi \dot{\lambda}=0
$$

be simultaneously satisfied at all times.

It is interesting to note that the above constitutive model shares a common structure with other rate-independent theories such as classical plasticity. In this latter case, a principal objective of the theory is to predict the evolution of the plastic strains while, in the case at hand, interest is focused on the evolution of the effective elastic moduli. In spite of these similarities, certain aspects of the behavior of materials undergoing microcracking do not have a counterpart in plasticity. The effect of closure of microcracks under load reversal falls within that category. By this mechanism microcracks can become inactive and cease to contribute to the flexibility of the material. Consideration of this effect requires adding further structure to the model. Microcrack closure can be modelled within a phenomenological theory as a unilateral constraint which requires that the deformation contributed by the microcracks be always tensile in all directions (Ortiz, 1985). In the present study attention is confined to monotonic loading processes for which microcrack closure is of no concern.

For the purpose of the asymptotic analysis that follows it proves more convenient to employ a deformation type con-

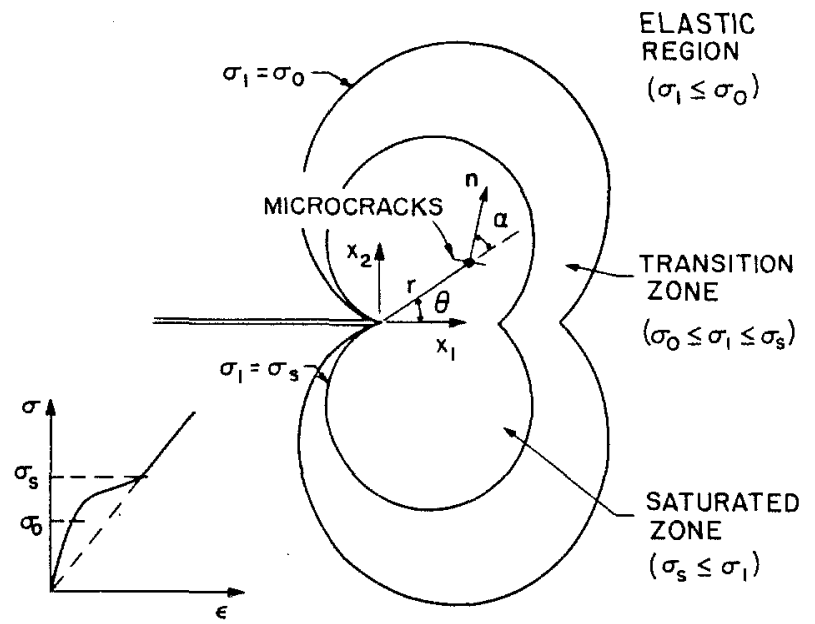

Fig. 2 Small scale microcracking problem for stationary crack

stitutive theory rather than the incremental model introduced above. A deformation theory of damage can be readily obtained by integrating the incremental constitutive equations along proportional stress paths. This results in the following stress-strain relation

$$
\epsilon_{i j}=\left(C_{i j k l}^{o}+\lambda\left(\sigma_{1}\right) n_{i} n_{j} n_{k} n_{l}\right) \sigma_{k l}
$$

where $C_{i j k l}^{o}$ are the isotropic elastic moduli of the uncracked material, $\mathbf{n}$ is the direction of maximum tensile stress and the function $\lambda\left(\sigma_{1}\right)$ can be determined directly from the uniaxial tension test. A typical uniaxial tension stress-strain curve is shown in Fig. 1 (Fu, 1983). It is seen that the material initially exhibits an elastic domain after which damage starts to accumulate. Eventually, a saturation stage is reached wherein no further damage takes places. In this saturation stage equation (6) simplifies to

$$
\epsilon_{i j}=\left(C_{i j k l}^{o}+\lambda_{s} n_{i} n_{j} n_{k} n_{l}\right) \sigma_{k l}=C_{i j k l}^{o} \sigma_{k l}+\lambda_{s} \sigma_{1} n_{i} n_{j}
$$

where $\lambda_{s}$ is a constant of value

$$
\lambda_{s}=\frac{1}{E_{s}}-\frac{1}{E_{o}}
$$

Here, $E_{o}$ is the initial Young's modulus of the material and $E_{s}$ is the slope of the uniaxial tension stress-strain curve in the saturation range.

The stress-strain relation (6) has a hyperelastic structure

$$
\epsilon_{i j}=\frac{\partial \chi(\sigma)}{\partial \sigma_{i j}}
$$

where the complementary energy potential $\chi(\sigma)$ takes the form

$$
\chi(\sigma)=\frac{1}{2} C_{i j k l}^{o} \sigma_{i j} \sigma_{k l}+f\left(\sigma_{1}\right)
$$

The functions $f\left(\sigma_{1}\right)$ and $\lambda\left(\sigma_{1}\right)$ are related by $f^{\prime}=\lambda$. In the saturation stage equation (6) reduces to (7) and $f\left(\sigma_{1}\right)$ to $\lambda_{s} \sigma_{1}^{2} / 2$, which renders the complementary energy potential (10) a homogeneous function of degree two of the stress tensor. Therefore, the strain energy potential $W(\epsilon)$ is also a homogeneous function of degree two and satisfies the identity

$$
W(\epsilon)=\chi(\sigma)=\frac{1}{2} \sigma_{i j} \epsilon_{i j}, \text { when } \epsilon_{i j}=\frac{\partial \chi(\sigma)}{\partial \sigma_{j}}
$$

i.e., the strain and complementary energy potentials take the same numerical value when evaluated at stresses and strains which satisfy the stress-strain relations (9).

\section{Asymptotic Fields for a Stationary Crack}

Throughout this work it is assumed that the region around 
the crack tip to which microcracking is confined is small compared with the length of the crack but orders of magnitude larger than the characteristic size of the microcracks and their mean separation. Under the first condition an asymptotic problem can be formulated for a semi-infinite crack as shown in Fig. 2. The second condition is needed for the constitutive model presented above to apply. In this paper attention is confined to plane strain conditions and Mode I loading, i.e., applied loads which result in stress fields which are symmetric with respect to the plane of the crack.

Three well-differentiated regions surrounding the crack tip can be identified, Fig. 2. In the innermost region the strains are large enough so that the material can be assumed to be in the saturation stage. On the other hand, at points far away from the crack tip the material behavior is linear isotropic elastic and the state of stress is given by

$$
\sigma_{i j}(r, \theta)=\frac{K_{\infty}}{\sqrt{2 \pi r}} \tilde{\sigma}_{i j}^{o}(\theta)
$$

where $K_{\infty}$ is the remote stress-intensity factor and the universal angular distributions $\tilde{\sigma}_{i j}^{o}(\theta)$ for a linear isotropic elastic material are given by

$$
\left\{\begin{array}{c}
\tilde{\sigma}_{r r}^{o}(\theta) \\
\tilde{\sigma}_{\theta \theta}^{o}(\theta) \\
\tilde{\sigma}_{\theta \theta}^{o}(\theta)
\end{array}\right\}=\left\{\begin{array}{l}
(5 / 4) \cos (\theta / 2)-(1 / 4) \cos (3 \theta / 2) \\
(3 / 4) \cos (\theta / 2)+(1 / 4) \cos (3 \theta / 2) \\
(1 / 4) \sin (\theta / 2)+(1 / 4) \sin (3 \theta / 2)
\end{array}\right\}
$$

The value of $K_{\infty}$ depends upon the particular geometry of the cracked specimen and represents the influence of the applied loading. In between the inner and outer field lies a transition zone in which the material is partially saturated.

The behavior of the material surrounding the crack tip is assumed to be described by the stress-strain law (6). Since these relations derive from a complementary energy potential which is homogeneous of degree two, a classical argument (see, e.g., Rice 1968) shows that the leading term in the asymptotic expansion of the stress field has to be of the form

$$
\sigma_{i j}(r, \theta)=\frac{K_{t}}{\sqrt{2 \pi r}} \tilde{\sigma}_{i j}(\theta)
$$

where $K_{t}$ is the local stress-intensity factor of the near-tip fields and the angular distributions $\tilde{\sigma}_{i j}(\theta)$ are to be determined. In general $K_{t}$ is different from $K_{\infty}$ because the crack tip is shielded from the remote loads by the intervening microcracks. Assuming that crack growth is controlled by the value of $K_{t}$ it becomes of primary interest to determine the relation between $K_{t}$ and $K_{\infty}$.

In view of the fact that the constitutive behavior of the material surrounding the crack tip is nonlinear and strongly anisotropic one would expect singular stress fields which substantially depart from the linear isotropic solution. It is shown next that this is not the case. In fact, the linear isotropic stress field provides the exact asymptotic solution of the problem, i.e.,

$$
\tilde{\sigma}_{i j}(\theta)=\tilde{\sigma}_{i j}^{o}(\theta)
$$

Under certain circumstances, a similar situation is encountered in materials exhibiting linear creep and grain boundary cavitation (Hutchinson, 1983). On the basis of this observation, Hutchinson (1983) anticipated the result stated above, namely, that constitutive relation (7) implies the same angular distribution of stresses as in the linear elastic solution.

To prove equation (15) we start by noting that the stress field (14), (15) satisfies equilibrium and traction-free boundary conditions on the crack faces. Thus, it only remains to be shown that the corresponding strains satisfy compatibility. With reference to Fig. 2, the direction of maximum tensile stress is computed to be
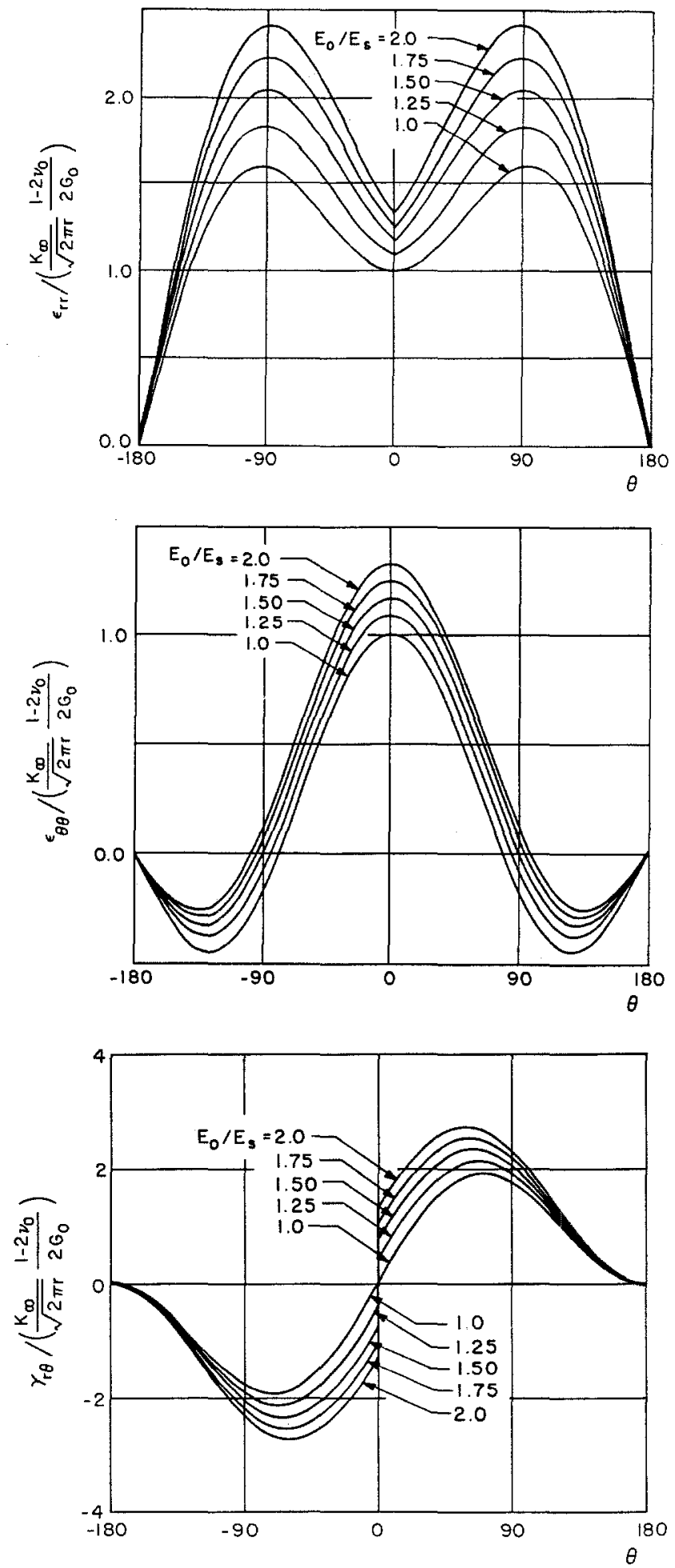

Fig. 3 Angular variations of the near-tip strain field $\left(\nu_{0}=0.25\right)$

$$
\mathbf{n}=(\cos \alpha, \sin \alpha), \quad \alpha= \begin{cases}\pi / 4-\theta / 4, & 0<\theta \leq \pi ; \\ -\pi / 4-\theta / 4, & -\pi \leq \theta<0 ;\end{cases}
$$

Thus, the angle $\alpha$ made by $\mathbf{n}$ and the radial direction varies linearly with the polar angle $\theta$ from a value of $\alpha=45^{\circ}$ at $\theta=0^{+}$ to $\alpha=0$ on the crack face $\theta=\pi$. The vector $\mathbf{n}$ can be regarded as giving an indication of the preferred orientation of the microcracks. In this light, it is interesting to note that for $\theta=0$ one has $\tilde{\sigma}_{r r}=\tilde{\sigma}_{\theta \theta}$ and $\tilde{\sigma}_{r \theta}=0$. This would appear to render $\alpha$ in- 
determinate on the plane of the crack. However, this indeterminacy can be resolved by computing the limiting values of $\alpha$ as the plane $\theta=0$ is approached from above and below. This operation yields two values of $\alpha= \pm 45^{\circ}$, respectively. Thus, the model predicts two families of perpendicular microcracks at $\theta=0$ symmetrically distributed with respect to the plane of the crack.

Substituting (16) into (7) the asymptotic strain field is computed to be

$$
\left\{\begin{array}{c}
\epsilon_{r r}(r, \theta) \\
\epsilon_{\theta \theta}(r, \theta) \\
\gamma_{r \theta}(r, \theta)
\end{array}\right\}=\frac{K_{t}}{\sqrt{2 \pi r}} \frac{1}{2 G_{o}}
$$

$$
\begin{aligned}
& \left\{\begin{array}{r}
\left(5 / 4-2 \nu_{o}\right) \cos (\theta / 2)-(1 / 4) \cos (3 \theta / 2) \cos (3 \theta / 2) \cos (\theta / 2)+(1 / 4) \sin (3 \theta / 2) \\
\left(3 / 4-2 \nu_{o}\right) \sin (\theta / 2)+(1 / 2) \sin (1 / 2) \\
\left(\frac{K_{t}}{\sqrt{2 \pi r}} \lambda_{s}\left(\cos \frac{\theta}{2}+\frac{1}{2} \sin \theta\right)\right.
\end{array}\right. \\
& \left\{\begin{array}{c}
(1+\sin (\theta / 2)) / 2 \\
(1-\sin (\theta / 2)) / 2 \\
\cos (\theta / 2)
\end{array}\right\}, 0<\theta \leq \pi
\end{aligned}
$$

where $E_{o}, \nu_{o}$, and $G_{o}=E_{o} / 2\left(1+\nu_{o}\right)$ are the initial Young's modulus, Poisson's ratio, and shear modulus of the uncracked material. It is noted that the first term is the isotropic linear elastic solution corresponding to a stress intensity factor $K_{\iota}$. The second term represents the effect of damage and vanishes identically for $\lambda_{s}=0$, i.e., $E_{s}=E_{0}$. The strains in the lower half plane $-\pi \leq \theta<0$ are obtained from the symmetry conditions

$$
\begin{array}{r}
\epsilon_{r r}(r,-\theta)=\epsilon_{r r}(r, \theta), \quad \epsilon_{\theta \theta}(r,-\theta)=\epsilon_{\theta \theta}(r, \theta), \\
\gamma_{r \theta}(r,-\theta)=-\gamma_{r \theta}(r, \theta)
\end{array}
$$

The computed angular distributions of the strain components are shown in Fig. 3 as function of the material parameters involved. A noteworthy feature of the solution is that the shearing strain $\gamma_{r \theta}$ exhibits a jump across the plane of the crack.

Lengthy but straightforward algebra shows that the strain field (17) identically satisfies the compatibility equation. Hence, equations (14), (15) do indeed provide a closed form asymptotic solution of the problem. The displacement field can be computed from the strain-displacement relations to obtain

$$
\begin{gathered}
\left\{\begin{array}{l}
u_{r}(r, \theta) \\
u_{\theta}(r, \theta)
\end{array}\right\}=\frac{K_{t}}{4 G_{o}} \sqrt{\frac{r}{2 \pi}} \\
\left\{\begin{array}{c}
\left(2 \kappa_{o}-1\right) \cos (\theta / 2)-\cos (3 \theta / 2) \\
-\left(2 \kappa_{o}+1\right) \sin (\theta / 2)+\sin (3 \theta / 2)
\end{array}\right\}+K_{t} \lambda_{s} \sqrt{\frac{r}{2 \pi}} \\
\left\{\begin{array}{c}
(\cos (\theta / 2)+(1 / 2) \sin \theta)(1+\sin (\theta / 2)) \\
\cos \theta-\sin (\theta / 2)-\sin ^{3}(\theta / 2)-1 \\
0<\theta \leq \pi
\end{array}\right\},
\end{gathered}
$$

where $\kappa_{o}=3-4 \nu_{o}$. The displacements in the lower half plane follow from the symmetry conditions

$$
u_{r}(r,-\theta)=u_{r}(r, \theta), \quad u_{\theta}(r,-\theta)=-u_{\theta}(r, \theta)
$$

Of particular interest is the crack opening profile

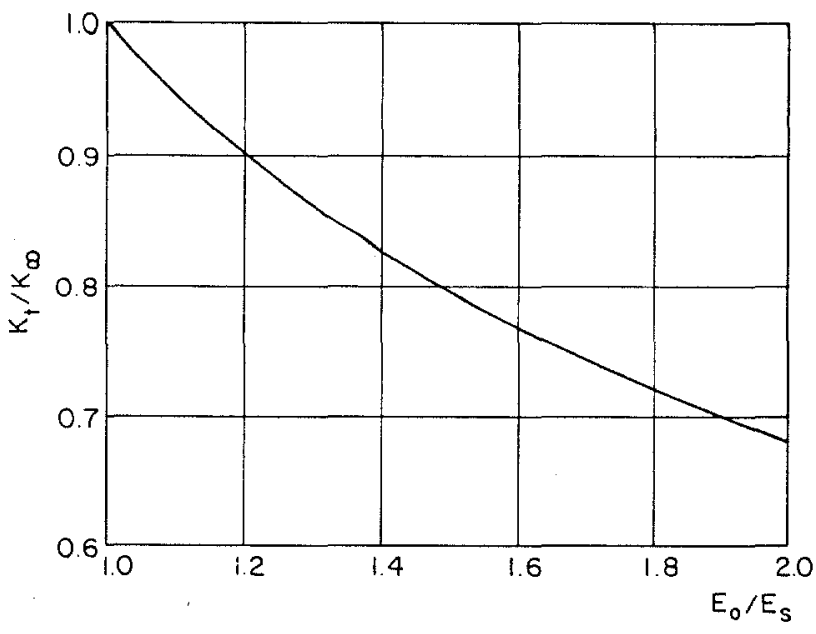

Fig. 4 Near-tip stress-intensity factor as a function of the extent of elastic degradation $\left(v_{0}=0.25\right)$

$$
\delta(r)=-u_{\theta}(r,-\pi)+u_{\theta}(r,-\pi)=8 K_{t}\left(\frac{1-\nu_{o}^{2}}{E_{o}}+\lambda_{s}\right) \sqrt{\frac{r}{2 \pi}}
$$

As can be seen, the opening profile is parabolic as in the linear elastic solution.

The asymptotic analysis presented above has been based on the deformation theory of damage given in Section 2. However, since the stress field about the crack tip is identical to the stress field in the outer undamaged region except for an amplitude factor it can be concluded that the stress paths at all material points are nearly proportional and the solution given above is consistent with the incremental constitutive model as well. A similar situation was encountered by Hutchinson (1968) and Rice and Rosengren (1968) who based their analysis on a deformation theory of plasticity to find a posteriori that their solution satisfies the incremental constitutive equations as well.

\section{Crack Tip Stress-Intensity Factor: Application of the $J$-Integral}

To have a complete asymptotic solution of the problem under consideration, it remains to determine the value of the crack tip stress-intensity factor $K$, as a function of the amplitude $K_{\infty}$ of the applied $K$ field. This relation follows simply from an application of the $J$-integral of Rice (Rice, 1968). It has been shown above that under monotonic loading the stress path at all points is nearly proportional and the response of the material is indistinguishable from that of a small strain, nonlinear elastic solid with complementary energy potential (10). Under these conditions, the formalism of the $J$-integral applies. Let us recall that

$$
J=\int_{\Gamma}\left[W(\epsilon) m_{1}-\sigma_{i j} m_{j} u_{i, 1}\right] d s
$$

Here, m signifies the outer normal to the contour $\Gamma$ encircling the crack tip. If the contour is chosen to lie entirely within the remote field, $J$ reduces to the classical expression

$$
J_{\infty}=\frac{1-\nu_{o}^{2}}{E_{o}} K_{\infty}^{2}
$$

For a contour shrunk down to the crack tip (22) can be evaluated from equations (14), (15), and (19). For the particular material model under consideration the strain and complementary energy potentials coincide in numerical value and $W(\epsilon)$ in equation (22) can be replaced by $\chi(\sigma)$ as computed from (7), (14), and (15). A lengthy but straightforward computation yields 


$$
J_{t}=\frac{1-v_{o}^{2}}{E_{o}} K_{t}^{2}+\beta \lambda_{s} K_{t}^{2}
$$

where the numerical constant $\beta$ takes the value $\beta=1.0942$. The path-independence of $J$ necessitates

$$
J_{t}=J_{\infty}
$$

from where one finds

$$
\frac{K_{t}}{K_{\infty}}=\frac{1}{\sqrt{1+\frac{\beta}{1-\nu_{o}^{2}}\left(E_{o} / E_{s}-1\right)}}
$$

The dependence of $K_{t} / K_{\infty}$ on $E_{o} / E_{s}$ is shown in Fig. 4. A substantial reduction in $K$ is observed even for moderate deviations from elastic behavior.

\section{Discussion}

The analysis presented above has been based on a model of damage in which permanent strains and rate effects are assumed to be negligible. The model incorporates some of the complexities that are encountered in most progressively fracturing materials such as a strong damage-induced elastic anisotropy. However, the constitutive framework is simple enough that a closed form analytical solution for the asymptotic problem can be obtained.

The computed results are indicative of a significant reduction in the crack tip stress-intensity factor from the remote $K$. Unfortunately, the net toughness enhancement cannot be expected to be as substantial as Fig. 4 would tend to suggest due to the fact that the microcracks created ahead of the main crack degrade the crack extension resistance of the material (Evans, 1984). The main microstructural mechanism underlying this latter effect is microcrack coalescence, a process which is poorly understood at present. Thus, it would appear that a detailed understanding of the toughness properties of ceramics will inevitably require further experimental and analytical research.

\section{Acknowledgments}

The author is indebted to helpful and stimulating conversations with C. F. Shih and J. W. Hutchinson. The support of the Office of Naval Research through grant N00014-85$\mathrm{K}-0720$ is gratefully acknowledged.

\section{References}

Budiansky, B., Hutchinson, J. W., and Lambropoulos, J. C., 1983, "Continuum Theory of Dilatant Transformation Toughening in Ceramics," Int. J. Solids Structures, Vol. 19, pp. 337-355.

Claussen, N., 1976, "Fracture Toughness of $\mathrm{Al}_{2} \mathrm{O}_{3}$ with an Unstabilized $\mathrm{ZrO}_{2}$ Dispersed Phase," J. Am. Ceram. Soc., Vol. 59, pp. 49-51.

Evans, A. G., 1984, "Aspects of the Reliability of Ceramics," Defect Properties and Processing of High-Technology Monmetallic Materials, Crawford, J. H., Chen, Y., and Sibley, W. A., eds., North-Holland, pp. 63-80.

Fu, Y., 1983, "Mechanics of Microcrack Toughening in Ceramics," Ph.D. Thesis, Unjversity of California, Berkeley, Calif.

Hoagland, R. G., Hahn, G. T., and Rosenfield, A. R., 1973, "Influence of Microstructure on the Fracture Propagation in Rock," Roc. Mech., Vol. 5, pp. 77-106.

Hoagland, R. G., and Embury, J. D., 1980, “A Treatment of Inelastic Defor" mation Around a Crack Tip Due to Microcracking," J. Am. Ceram. Soc., Vol. 63, pp. 404-410.

Horii, H., and Nemat-Nasser, S., 1983, "Overall Moduli of Solids with Microcracks: Load-Induced Anisotropy," J. Mech. Phys. Solids, Vol. 31, pp. 155-171.

Hutchinson, J. W., 1968, "Singular Behavior at the End of a Tensile Crack in a Hardening Material," J. Mech. Phys. Solids, Vol. 16, pp. 13-31.

Hutchinson, J. W., 1983, "Constitutive Behavior and Crack Tip Fields for Materials Undergoing Creep-Constrained Grain Boundary Cavitation," Acta Metall., Vol. 31, No. 7, pp. 1079-1088.

Kachanov, M., 1985, "A Simple Technique of Stress Analysis in Elastic Solids with Many Cracks," Int. J. Fracture, Vol. 28, pp. 11-19.

Ortiz, M., 1985, "A Constitutive Theory for the Inelastic Behavior of Con crete," Mechanics of Materials, Vol, 4, pp. 67-93.

Rice, J. R., 1968, "Mathematical Analysis in the Mechanjes of Fracture," Fracture, Liebowitz, H., ed., Vol. 2, Academic Press, pp. 191-311.

Rice, J. R., and Rosengren, G. F., 1968, "Plane Strain Deformation Near a Crack Tip in a Power Law Hardening Material," J. Mech. Phys. Solids, Vol. 16, pp. 1-12.

Wu, C. C., Freiman, S. W., Rice, R. W., and Mecholsky, J. J., 1978, "Microstructural Aspects of Crack Propagation in Ceramics," J. Mater. Sci., Vol. 13, pp. 2659-2670.

\title{
Readers Of
}

\section{The Journal Of Applied Mechanics Will Be Interested In:}

\author{
AMD-Vol. 75
}

\section{Computational Mechanics_Advances and Trends}

\author{
Editor: A. K. Noor
}

Presented at the ASME Winter Annual Meeting, Anaheim, California, December 7-12, 1986.

\begin{abstract}
The twenty-four papers contained in this volume document clearly the strides made in a number of aspects of computational mechanics, identify some of the anticipated industry needs in this area, discuss the opportunities provided by new hardware and parallel algorithms, and outline some of the current government programs in computational mechanics. The topic headings in the symposium are largely represented by the section headings of this volume, namely Opportunities Provided by New Hardware and Parallel Algorithms, Industry Needs and Applications of Computational Mechanics, Recent Advances in Computational Structural and Solid Mechanics, Recent Advances in Computational Fluid Dynamics, Future Directions of Commercial Software Systems, and Government Programs in Computational Mechanics.
\end{abstract}

\section{Bk. No. H00344 492pp. \$70 list \$56 ASME Members}

Descriptions of other volumes of interest appear on pages $92,104,120,126,189,208,214,225$, and 242. 